\title{
公HAD
}

ISSN-L: 2530-5115

DOI: http://doi.org/10.22585/hospdomic.v2i1.34

\section{Transfusiones sanguíneas Domiciliarias en Pacientes Paliativos}

\author{
Home Blood Transfusions in Palliative Patients
}

Catalina Cases Alarcón, Nieves Santacruz Carmona

Hospital General Universitario de Alicante. Instituto de Investigación Sanitaria y Biomédica de Alicante ISABIAL-FISABIO, Alicante, España.

Correspondencia/Correspondence

Catalina Cases Alarcón

cathycases@ono.com

Recibido/Received

04.12 .2017

Aceptado/Accepted

12.01 .2018
Conflicto de Intereses/Competing interest No existe ningún conflicto de interés en el presente estudio.

CÓMO CITAR ESTE TRABAJO | HOW TO CITE THIS PAPER

Cases Alarcón C, Santacruz Carmona N. Transfusiones sanguíneas Domiciliarias en Pacientes Paliativos. Hosp Domic. 2018;2(1):19-25 


\section{RESUMEN}

Introducción: La asistencia a los pacientes crónicos complejos ocupa gran parte de la actividad asistencial de las Unidades de Hospitalización a Domicilio (HAD), en los que son frecuentes los cuadros de anemia crónica que precisan ocasionalmente transfusiones por clínica secundaria al déficit de la capacidad de oxigenación de los tejidos por falta de aporte.

Objetivo: Conocer la prevalencia de transfusiones y las características de los pacientes en la HAD del Hospital General Universitario de Alicante.

Material y Método: Estudio descriptivo transversal de los pacientes transfundidos en la HAD del Hospital Universitario General de Alicante desde el 1 de Octubre de 2016 hasta el 31 de marzo de 2017.

Resultados: Se realizaron 136 transfusiones a 52 pacientes con una mediana de 82 años IIQ [68-88] de los que 53,8\% eran varones. Fueron mayoritariamente paliativos. La Hemoglobina media con la que se transfundieron fue de 7,8 $\mathrm{g} / \mathrm{dl} \pm 0,9$ y todos presentaron sintomatología. No se registró ningún tipo de reacción adversa. El grado de satisfacción de los pacientes y familiares fue de elevado $\bar{x} 9,7 \pm 0,3$. La prevalencia fue de 121,86 transfusiones /1000 pacientes en la HAD.

Conclusiones: Las transfusiones han sido un procedimiento seguro en el domicilio del paciente. La mayor parte de los pacientes que han recibido transfusiones eran paliativos. Todas las transfusiones realizadas estaban indicadas analítica y clínicamente. El elevado grado de satisfacción de pacientes y familiares hace inferir que se está contribuyendo a mejorar la calidad de vida.

Palabras clave: Servicios de Atención a Domicilio Provisto por Hospital; Transfusión Sanguínea; Transfusión de Componentes Sanguíneos; Anemia.

\section{ABSTRACT}

Introduction: Assistance of complex chronic patients occupies much of the health care activity of home care hospital bassed $(\mathrm{HCHB})$. The chronic anemias are frequent as a secondary clinic picture due to the deficit of the tissues oxygenation capacity for lack of contribution.

Aim: to know the prevalence of transfusions and the characteristics of the patients in the $\mathrm{HCHB}$ of the Hospital General Universitario de Alicante.

Material and methods: Descriptive, cross-sectional study of transfused patients in the $\mathrm{HCHB}$ of General University of Alicante from the 1st of October 2016 until the 31th of march of 2017

Results: 136 transfusions were performed in 52 patients with a median of 82 years IQ [68-88]. The most of them were palliatives and the $53,8 \%$ were males.

They were transfused using an average hemoglobin of $7,8 \mathrm{~g} / \mathrm{dl} \pm 0,9$ and all of them showed symptomatology. No type of adverse reaction was registered.

The degree of patient satisfaction and their relatives was high. $\bar{x} 9.7 \pm 0.3$. The prevalence was 121.86 transfusions / 1000 patients in the HAD.

Conclusions: The transfusions have been a safe procedure at the patient's home. The most part of the transfused patients were palliatives. All the recorded transfusions were indicated analytical and clinically. The high degree of patients satisfaction and their relatives makes infer that we are contributing to improve their quality of life.

Keywords: Home Care Services, HospitalBased; Blood Transfusion; Blood Component Transfusion; Anemia. 


\section{INTRODUCCIÓN}

Las transfusiones de sangre son un procedimiento médico por el cual un paciente recibe sangre o alguno de sus componentes por vía intravenosa. Están indicadas en aquellos pacientes que presentan una falta de componentes sanguíneos y que no tienen otra alternativa de corregirlos.

La anemia constituye una de las patologías o comorbilidades más prevalentes, especialmente en población de edad avanzada (17-63\%) $(1,2)$. Es una de las principales causas de necesidad de ingreso hospitalario e incremento de la estancia hospitalarias, deterioro de la calidad de vida y mortalidad. Según la definición de los criterios de la OMS, es común en las personas mayores que viven en la comunidad y es particularmente común en los residentes de hogares de ancianos y en aquellos que ingresan en el hospital. (1).

En las Unidades de Hospitalización a Domicilio (HAD), la asistencia de los pacientes crónicos complejos ocupa gran parte de la actividad asistencial. En estos pacientes son frecuentes los cuadros de anemia crónica, y las transfusiones están indicadas cuando la anemia es sintomática y refractaria a otros tratamientos etiológicos, manteniendo un déficit en la capacidad de oxigenación de los tejidos por falta de aporte. Habitualmente se emplea el valor de hemoglobina (hb) como indicador tanto de la masa de hematíes como de la liberación de $\mathrm{O} 2$, aunque no está perfectamente definido el valor de hb por debajo del cual, se comprometa la liberación de oxígeno y se manifieste la hipoxia tisular, por lo menos en situaciones de anemia crónica $(3,4)$. Por lo que la decisión de la indicación se basa en parámetros indirectos (saturación de oxígeno, etc.) considerando el umbral transfusional en pacientes normovolémicos sin patología cardiovascular en torno a $7 \mathrm{~g} / \mathrm{dl}$ de hb (4-6). En estos casos, las transfusiones de eritrocitos son necesarias para aumentar la capacidad de transporte de oxígeno de la sangre, incrementando de esta manera la concentración de hemoglobina. Sin embargo, no hay consenso sobre las indicaciones precisas para su uso, a pesar de los numerosos intentos en forma de directrices y los acuerdos alcanzados en las Conferencias de Consenso (7). Los síntomas de la anemia crónica dependen de la edad del paciente, el nivel de actividad y los problemas médicos coexistentes, como las enfermedades cardiovasculares, respiratorias, hematológicas malignas y tumores sólidos entre otras. Los síntomas más frecuentes son la fatiga y la disnea, incluso pueden aparecer dolores precordiales, algunos de estos síntomas pueden ser subjetivos, pero siguen siendo útiles para determinar la necesidad de transfusión de glóbulos rojos en pacientes con anemia crónica. En una revisión sistemática que incluye 12 estudios de pacientes con cáncer avanzado con anemia para identificar si la transfusión de sangre es un tratamiento útil para ellos. Estos estudios indicaron que la fatiga y la disnea mejoraron inmediatamente después de la transfusión, pero esta mejoría comenzó a disminuir después de un período de dos semanas. De los pacientes que recibieron una transfusión del $31 \%$ al $70 \%$ presentó un beneficio transitorio que se prolongó alrededor de dos semanas (8).

En la anemia crónica se desarrollan una serie de mecanismos adaptativos que la hacen mejor tolerada que la anemia aguda, debido a una mejor administración de oxígeno asociada con un aumento de 2,3-difosfoglicerato (DPG) y un cambio en la curva de disociación de oxígeno. La reserva de capacidad de transporte de oxígeno es tal, que el gasto cardíaco en reposo no suele aumentar hasta que la concentración de hemoglobina cae por debajo de $7 \mathrm{~g} / \mathrm{dl}$.

Es notable el beneficio que las HAD han proporcionado a estos pacientes permitiendo su tratamiento en el domicilio. Hay que tener en cuenta el perfil de estos pacientes que tienen disminución de la capacidad funcional, una movilidad reducida y gran dependencia, todo ello condiciona grandes dificultades para su traslado hasta el hospital. La asistencia de estos pacientes en la HAD conlleva además de la realización de la analítica previa y de la indicación de la pertinencia de la transfusión, una atención integral y multidisciplinar. Para ello es necesaria una buena coordinación con el Servicio de Banco de sangre, lo que permite que se realice el procedimiento en el mismo día. 
En este trabajo se propone revisar las transfusiones realizadas por una HAD durante un semestre de actividad, para conocer la prevalencia y las características de los pacientes y del procedimiento.

\section{MATERIAL Y MÉTODO}

Estudio transversal de los pacientes transfundidos en la Unidad de Hospitalización a Domicilio del Hospital Universitario General de Alicante desde el 1 de Octubre de 2016 hasta el 31 de marzo de 2017.

Criterios de inclusión: pacientes de cualquier grupo patológico que precisara transfusión de sangre durante su ingreso en la HAD, que estuviese en Seguimiento programado o mediante interconsulta de otro Servicio con este motivo. No se aplicó ningún criterio de exclusión.

Los datos se obtuvieron del registro de la historia clínica de enfermería. El grupo patológico se registró como; paliativo oncológico, paliativo no oncológico y no paliativo. Para el cálculo de la prevalencia se estimaron el número de altas realizadas en la HAD durante ese periodo siendo de 1116 pacientes. El grado de adecuación de la indicación de la transfusión (bien indicada / mal indicada) se determinó por el nivel de hemoglobina y la presencia de síntomas. Para determinar el grado de satisfacción de los pacientes con la asistencia sanitaria recibida en su domicilio, se utilizó una escala analógico visual graduada desde 0 = muy insatisfactoria hasta 10 = muy satisfactoria.

Para el análisis de los datos se utilizaron técnicas descriptivas mediante el cálculo de las frecuencias absolutas y relativas (porcentajes) de las variables cualitativas y en el caso de las cuantitativas se emplearon medidas de tendencia central, y de dispersión de los datos (desviación estándar SD o intervalo intercuartílico IIQ).

El control de calidad de los datos se efectuó a de dobles tablas y búsqueda activa de errores, cuando fueron encontrados se corrigieron mediante la consulta con la fuente original de los datos.

Para el análisis se utilizó el paquete estadístico Statistical Packageforthe Social Sciences SPSSR 22.0 .

\section{RESULTADOS}

Se han realizado 136 transfusiones a 52 pacientes $(\bar{x} 2,62 \pm 3,07)$ con una mediana de edad 82 años IIQ [68-88] de los que 53,8\% eran varones. La prevalencia fue de 121,86 transfusiones /1000 pacientes en la HAD.

El tipo de paciente que recibieron transfusión (ver figura 1) fueron con mayor frecuencia los clasificados como "oncologicos paliativos" 26 (50\%) seguidos de los "paliativos no oncológicos" $14(26,9 \%)$

La Hemoglobina media con la que se transfundieron fue de 7,8 $\mathrm{g} / \mathrm{dl} \pm 0,9$. Todos los pacientes presentaron sintomatología previa a la transfusión, siendo el síntoma predominante la debilidad extrema (ver figura 2), por lo tanto el 100\% de las transfusiones estaban correctamente indicadas.

Los pacientes presentaban un buen estado cognitivo $82,7 \%$ y $7,7 \%$ demencia leve-moderada, en el 9,6\% no se registró el dato. Recibieron concentrado de hematíes el 90,4\%, plaquetas 1,9\% y ambos el $7,7 \%$. No se registró ningún tipo de reacción adversa. El grado de satisfacción de los pacientes y familiares fue de $\bar{x} 9,7 \pm 0,3$. 
Fig. 1. Tipo de pacientes

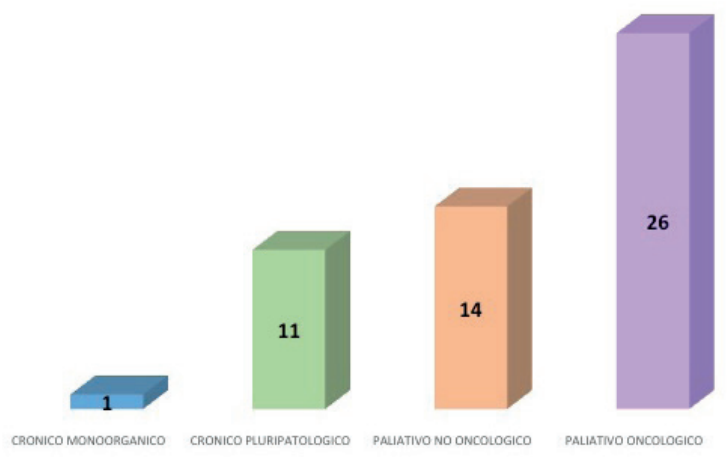

Fig. 2. Síntomas presentados antes de la transfusión

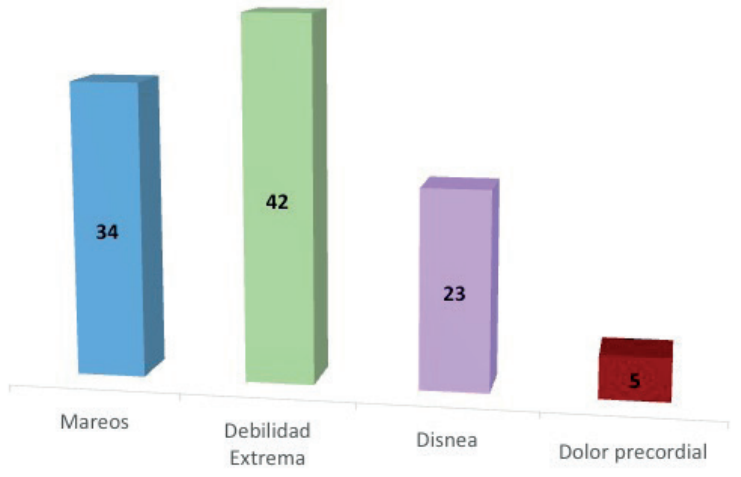

\section{DISCUSIÓN}

Este trabajo es una de las mayores series recogidas de pacientes con transfusión de hemoderivados en hospitalización a domicilio. Habitualmente los trabajos científicos consultados se refieren a las transfusiones dentro de la descripción de otros tratamientos o intervenciones que se han realizado en las Unidades de Hospitalización a Domicilio con un número muy escaso. Kodama y cols. (9), en 2009, en un estudio con intervención de 10 unidades de hospitalización a domicilio de 8 localidades diferentes, de 580 pacientes ingresados en las HAD realizan tan solo 3 transfusiones en 15 pacientes con enfermedad hematológica maligna con Performance Status (PS) 3-4, sin que aporten más datos sobre las mismas.

García Sanchez y cols. (10) en 68 pacientes ingresados en HAD del Hospital Marqués de Valdecilla durante el año 2013 realizan 216 transfusiones de concentrado de hematíes. En esta serie, 
la edad de los pacientes coincide con la del presente trabajo, aunque no especifican el estadio evolutivo del paciente, hablan de "enfermedad hematológica" en casi el 50\% de los casos y neoplasia sólida en el $22,2 \%$ con lo que podemos aproximar que las poblaciones son bastante similares. Declaran que un $13,7 \%$ de las transfusiones tenían una indicación dudosa o no conveniente, esta condición en el presente trabajo no se ha observado, estando todas las transfusiones indicadas.

No se ha registrado ninguna reacción adversa a pesar de que no se utilizó medicación pretransfusional. La satisfacción de pacientes y familiares nos hacen inferir que este procedimiento puede estar contribuyendo al aumento de la calidad de vida en esta etapa evolutiva tan compleja. A pesar de que la calidad de vida no se ha evaluado objetivamente en este trabajo, consideramos que sería una recomendación importante para tener en cuenta en estudios futuros con este tipo de población.

Preston y cols. (2012) (8) ante la incertidumbre del posible beneficio que la transfusión de sangre pueda proporcionar a los pacientes con cáncer avanzado y para responder a ¿quién presenta mayores probabilidades de mejoría y por cuánto tiempo?, plantearon una revisión sistemática en la que no encontraron ensayos clínicos e identificaron 12 estudios de intervención «antes y después» con 653 participantes que mostraron una tasa de mejoría subjetiva del $31 \%$ al $70 \%$. Cinco de los estudios evaluaron específicamente la mejoría de la fatiga mediante unas de escalas de fatiga observando una mejoría inmediata después de la transfusión que comenzó a disminuir sobre el día 14 después de la misma. Encontrando resultados similares para la disnea. En el presente estudio no se han evaluado objetivamente la mejoría de los síntomas, reconociendo este hecho como una limitación.

Conclusiones: La prevalencia fue de 121,86 transfusiones /1000 pacientes en la HAD.

Las transfusiones han sido un procedimiento seguro en el domicilio del paciente.

La mayor parte de los pacientes que han recibido transfusiones eran paliativos.

Todas las transfusiones realizadas estaban indicadas analítica y clínicamente.

El elevado grado de satisfacción de pacientes y familiares hace inferir que se está contribuyendo a mejorar la calidad de vida.

\section{BIBLIOGRAFÍA}

1. Gaskell H, Derry S, Moore RA, McQuay HJ. Prevalence of anaemia in older persons: systematic review. BMC Geriatrics. 2008;8(1):1-8. DOI: 10.1186/1471-2318-8-1; PMID: 18194534

2. McClellan W, Aronoff SL, Bolton WK, Hood S, Lorber DL, Tang KL, et al. The prevalence of anemia in patients with chronic kidney disease. Curr Med Res Opin. 2004;20(9):1501-10. DOI: 10.1185/030079904X2763; PMID: 15383200

3. Gramm J, Smith S, Gamelli RL, Dries DJ. Effect of transfusion on oxygen transport in critically ill patients. Shock. 1996;5(3):190-3. PMID: 8696982

4. Napolitano LM, Kurek S, Luchette FA, Anderson GL, Bard MR, Bromberg W, et al; EAST Practice Management Workgroup; American College of Critical Care Medicine (ACCM) Taskforce of the Society of Critical Care Medicine (SCCM). Clinical practice guideline: red blood cell transfusion in adult trauma and critical care. J Trauma. 2009;67(6):1439-42. DOI: 10.1097/ TA.0b013e3181ba7074; PMID: 20009700

5. Liumbruno G, Bennardello F, Lattanzio A, Piccoli P, Rossetti G. Recommendations for the transfusion of red blood cells. Blood Transfus. 2009;7(1):49-64. DOI: 10.2450/2008.0020-08; PMID: 19290081 
6. Pape A, Stein P, Horn O, Habler O. Clinical evidence of blood transfusion effectiveness. Blood Transfus. 2009;7(4):250-8. DOI: 10.2450/2008.0072-08; PMID: 20011636

7. Murphy MF, Wallington TB, Kelsey P, Boulton F, Bruce M, Cohen H, et al; British Committee for Standards in Haematology, Blood Transfusion Task Force. Guidelines for the clinical use of red cell transfusions. Br J Haematol. 2001; 113(1):24-31. PMID: 11328275

8. Preston N, Hurlow A, Brine J, Bennett M. Blood transfusions for anaemia in patients with advanced cancer. 2012;(2):CD009007. DOI: 10.1002/14651858.CD009007.pub2; PMID: 22336857

9. Kodama Y1, Takita M, Kawagoe S, Hirahara S, Kimura Y, Onozawa S, et al. Retrospective study on home care for patients with hematologic malignancies. Jpn J Clin Oncol 2009; 39(9)606-11. DOI: 10.1093/jjco/hyp064; PMID: 19535385

10. García Sánchez D, Neila Calvo S, Sanroma Mendizábal P, Aguilera Zubizarreta A, Sgaramella G, González C, et al. Transfusiones de Hemoderivados en Hospitalización a Domicilio (HAD). ¿Hacemos un uso óptimo de la sangre? Rev Clin Esp. 2014;214(Espec Congr):1136. 